\title{
Making an imprint on blood glucose monitoring
}

\author{
George S. Wilson
}

Because of its importance in monitoring blood glucose in type I insulin-dependent diabetics, a viable glucose sensor has come to represent perhaps the ultimate achievement in biosensor development. Despite considerable efforts to develop enzyme-based sensors and noninvasive techniques, a cheap and reliable glucose sensor remains some way off. In this issue, Frances Arnold and colleagues' report a glucopyranoside-imprinted polymer for measuring changes in proton concentration that may make possible the design of cheap and simple glucose-sensing devices in the future.

The pressing need for new, more reliable glucose sensors has been emphasized by a recent 10-year study-the Diabetes Control and Complications Trial $(\mathrm{DCCT})^{2}$-by the US National Institutes of Health (Bethesda, MD). This study clearly established that tight control of blood glucose reduces the long-term complications of diabetes by $30-70 \%$. Unfortunately, the benefit was also accompanied by a $300 \%$ increase in the incidence of hypoglycemia, a condition that can lead to loss of consciousness and one that diabetics wish specifically to avoid. Continuous monitoring of blood glucose via a biosensor would be an obvious solution. In such a scenario, monitoring would lead to activation of a hypoglycemic alarm-which would inform the patient of the onset of hypoglycemia-and ultimately trigger a pump capable of delivering the requisite amount of insulin.

The search for an effective glucose sensor that fulfills the above requirements has focused on three broad areas: noninvasive, invasive, and minimally invasive approaches. The first category includes techniques that rely on near-infrared ${ }^{3,4}$ and optical rotation measurements. Although the noninvasive nature of these approaches makes them attractive, they have not yet demonstrated the necessary reliability; indeed, it is not entirely clear that they are actually measuring glucose.

Invasive approaches involve long-term intravascular or intraperitoneal implantation

George S. Wilson is Higuchi professor of chemistry and pharmaceutical chemistry, department of chemistry, University of Kansas, Lawrence, KS 66045

(wilson@kuhub.cc.ukans.edu) of the detection device. On the whole, such systems are still not reliable enough-they should function for at least six months-to justify the discomfiture of surgical implantation. To date, the longest period for continued efficient function of an enzyme-based sensor is three months after implantation ${ }^{4}$.

Minimally invasive devices offer the most immediate, but by no means perfect, solution to continuous glucose monitoring. Such devices include the needle-type glucose oxidase-based sensors, which are implanted subcutaneously,6, and reverse iontophoresis, where the glucose is extracted across the skin and then detected with a glucose electrode? These minimally invasive devices only require
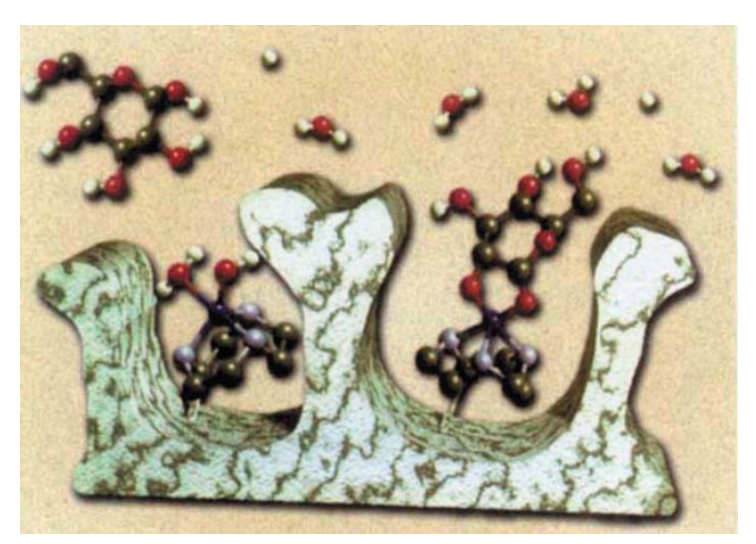

Figure 1. A glucopyranoside imprinted polymer.

a useful lifetime of 1-2 weeks, as they can be easily replaced without surgical intervention.

The ongoing search for a sensor that is more accurate, more reliable and cheaper to manufacture than the systems described above has prompted Arnold and colleagues to investigate a new avenue for glucose monitoring. Their system involves the binding of glucose to a tetraazacyclononane (TACN)- $\mathrm{Cu}^{2+}$ chelate, resulting in the displacement of water and hydroxide from the chelate, with the net release of a proton.

The concept of measuring glucose concentration by $\mathrm{pH}$ change is not new-it has been used previously to monitor the oxidation of glucose by oxygen catalyzed by glucose oxidase. In the past, the approach has been unsuccessful because the observed $\mathrm{pH}$ change is dependent not only on the rate of the enzymatic reaction, but also on the buffering capacity of the plasma, which can vary, depending on the levels of serum proteins at physiological $\mathrm{pH}$. The TACN$\mathrm{Cu}^{2+}$-glucose system may avoid this problem because measurements are made at highly alkaline $\mathrm{pH}$ values $(>\sim 10.8)$, where the buffering capacity both from proteins and from carbonate is low.

The fact that copper complex operates optimally at values of $\mathrm{pH} 11$ or greater does, however, raise the significant problem of how the (TACN) $-\mathrm{Cu}^{2+}$ chelate can be used to monitor glucose in plasma at physiological $\mathrm{pH}$ (7.3). One possible solution would be to use a negatively charged membrane $\left(\mathrm{Nafion}^{8}\right)$ to separate the alkaline environment of the sensor from the external solution at physiological $\mathrm{pH}^{9}$. It would clearly be simpler, however, if binding could be detected as a spectroscopic, perhaps fluorescence, change rather than by evolution of protons. For example, a glucose sensor based on the competitive binding of fluorescent-labeled dextran by a lectin (concanavalin A) inside a hollow fiber has been reported ${ }^{10}$ and, more recently, implants have been proposed that fluoresce when they bind glucose, providing readouts that can be measured optically through the skin.

What is potentially intriguing about the report from Arnold's laboratory is the claim that molecular imprinting enhances the selectivity of glucose recognition by the $\mathrm{TACN}-\mathrm{Cu}^{2+}$ complex-a factor that will be critical to application of the system as a practical sensor. The authors speculate that the polymer matrix may enhance selectivity by excluding endogenous molecules, which might otherwise interfere with glucose monitoring. As molecular imprinting is still in its infancy as a technique, it is difficult to predict the extent to which this approach can replace more classical solutions. The major challenge in this case, however, remains the development of sensors that can yield reliable and stable estimates of glucose concentration when exposed for extended periods of time (hours to days) to biological fluids. This seemingly simple goal has so far remained elusive.

1. Chen, G., et al, 1997. Nature Biotechnology 15:354-357.

2. Diabetes Complications and Control Trial Research Group. 1993. New Engl. J. Med. 329:977-986.

3. Small, G.W., Arnold, M.A., and Marquardt, L.A. 1993. Anal. Chem 65:3279-3289.

4. Robinson, M.R. et al. 1992. Clin. Chem. 38:1618-1622.

5. Gilligan, B.J., Shults, M.C., Rhodes, R.K., and Updike, S.J. 1994. Diabetes Care 17:882-887.

6. Johnson, K.W. et al. 1993. Biosens. Bioelectron. 7:709-714.

7. Poitout, V., Moatti-Sirat, D., Reach, G., Zhang, Y., Wilson, G.S., Lemonnier, F., and Klein, J.C. 1993. Diabetologia 36:658-663.

8. Tamada, J.A., Bohannon, N.J.V., and Potts, R.O. 1995. Nature Med. 1:1198.

9. Bindra, D.S. and Wilson, G.S. 1989. Anal. Chem. 61:2566-2570.

10. Schultz, J.S., Mansouri, S., and Goldstein, I.J. 1982. Diabetes Care 50:245-253. 